\title{
Estudio comparativo de agujas con sistema de seguridad vs agujas sin sistema de seguridad
}

\author{
Mónica Brazalet Tejerina ${ }^{1}$ - Beatriz Rico García ${ }^{2}-$ M $^{a}$ Luz Sánchez Tocino ${ }^{3}$ - Miriam Lentijo Noval ${ }^{1}$ \\ Alberto Sánchez Martín ${ }^{2}$ - Silvia Villoria González ${ }^{3}$
}

${ }^{1}$ Centro "Los Pinos" .F.R.I.A.T. Medina del Campo" - 2 Centro "El Castañar". F.R.I.A.T. Bejar

3 Centro "Las Encinas". F.R.I.A.T. Ciudad Rodrigo

\section{Resumen}

La práctica diaria de la enfermera en hemodiálisis implica riesgos laborales como las punciones accidentales, con el consecuente riesgo de contacto con agentes biológicos, Por tanto, se debe utilizar el mayor número posible de útiles con dispositivos de seguridad.

Nuestro objetivo fue evaluar las posibles diferencias entre las agujas con dispositivo de seguridad y las agujas sin dispositivo de seguridad para la punción de las fístulas arteriovenosas.

Llevamos a cabo un estudio prospectivo experimental con 42 pacientes portadores de fístula antóloga. Se creó un registro de datos relacionados con el "paciente", el "profesional" y el "material" para valorar las diferencias existentes entre ambos tipos de aguja. Se analizaron en un total de 35 pacientes en 455 sesiones

No hubo diferencias significativas en el uso o no del dispositivo de seguridad en cuanto al dolor provocado, los flujos de sangre, las presiones venosas o el sangrado de las punciones. De 910 punciones con

\begin{tabular}{|c|}
\hline Correspondencia: \\
Mónica Brazalez Tejerina \\
Centro de Hemodiálisis "Los Pinos" F.R.I.A.T \\
c/ Angustias 37 \\
47400 Medina del Campo. Valladolid \\
lospinos@friat.es
\end{tabular}

agujas con dispositivo de seguridad en 24 ocasiones se atascó el dispositivo.

PALABRAS CLAVE:

- FÍSTULA ARTERIOVENOSA INTERNA

- AGUJA

- DISPOSITIVO SEGURIDAD

- PUNCIÓN ACCIDENTAL

\section{Comparative study of safety needles and non-safety needles}

\section{Abstract}

The daily practice of nurses working in haemodialysis involves risks such as accidental injections, with the consequent risk of contact with biological agents. Accordingly, the highest possible number of tools with safety devices should be used.

Our aim was to evaluate the possible differences between needles fitted with safety devices and needles which did not have such devices in the puncture of arteriovenous fistulas.

We carried out an experimental prospective study with 42 patients who had antologous fistulas. A register was created of data relating to the "patient", the "professional" and the "material" in order to evaluate differences between the two 
different types of needle. A total of 35 patients were analysed over 455 sessions.

There were no significant differences in the use or caused, blood flow, venous pressure or bleeding on puncture. Of 910 injections with safety needles, the device jammed on 24 occasions

\section{KEY WORDS}

- INTERNAL ARTERIOVENOUS FISTULA

- NEEDLE

SAFETY DEVICE

ACCIDENTAL INJECTION not of the safety device with regard to the pain

zaba la incidencia de los accidentes que sufren diariamente los profesionales sanitarios con material punzante o cortante (jeringuillas, lancetas, etc) así como sus consecuencias reales. Habría que destacar el apartado de las enfermeras de diálisis ya que de los 16.374 casos que se declararon en 4 años (1996-2000), las matronas fueron el colectivo que más riesgo presentó ( 9 exposiciones /100matronas), pero las enfermeras de diálisis también presentaron un alto riesgo de exposición (3/100enfermeras hemodiálisis).

La Orden anteriormente mencionada que se basa originariamente en la Ley 31/1995, de 8 de noviembre, sobre Prevención de Riesgos Laborales, contempla una serie de requisitos y condiciones mínimas que deben tener las agujas de seguridad para fístulas arteriovenosas. Son las siguientes:

- Su estructura tendrá como fin primordial la eliminación de objetos punzocortantes.

La práctica de la hemodiálisis requiere el abordaje al sistema vascular de forma reiterada y segura. Esto conlleva, en nuestro quehacer diario, unos riesgos de accidentes relacionados con agentes biológicos, preferentemente provocados por punciones accidentales.

La Comunidad de Madrid ha publicado recientemente la Orden 827/2005 de 11 de mayo, en la que se establece e implantan los procedimientos de seguridad y el sistema de vigilancia frente al accidente con riesgo biológico en el ámbito sanitario de la Comunidad de Madrid. Y se ha establecido un plazo no superior a un año para introducir estos nuevos dispositivos que sustituirán de forma completa a los dispositivos tradicionales menos seguros. A pesar de que en nuestra Comunidad Autónoma no existe una orden legislativa similar, creemos interesante evaluar las diferencias existentes entre agujas con o sin dispositivo de seguridad y promover el uso de los primeros para disminuir los riesgos laborales en nuestro ámbito sanitario. Ya en el año 2005 se presentó el "Estudio Multicéntrico sobre las características de las Exposiciones a Riesgo Biológico Hemático de los Profesionales Sanitarios - Epinetac ", realizado por la Sociedad Española de Medicina Preventiva, Salud Pública e Higiene y el Consejo General de Enfermería de España. En dicho trabajo se anali-
- El sistema de seguridad no comprometerá en ningún caso la salud del paciente.

- El mecanismo de seguridad debe estar integrado en el dispositivo.

- La activación del mecanismo de seguridad habrá de manifestarse al usuario mediante una señal auditiva, táctil o visual. Y se realizará por el profesional sanitario, siempre que sea posible, con una mano.

- El mecanismo de seguridad no podrá ser desactivado y mantendrá su actividad protectora hasta que el dispositivo esté depositado en un contenedor de objetos punzocortantes.

- El dispositivo de seguridad habrá de ser fácil de utilizar, práctico, fiable y eficaz para alcanzar su finalidad. Siendo además compatible con otros accesorios que puedan utilizarse.

En tres centros periféricos de Castilla y León desde hace tiempo estamos utilizando agujas con dispositivos de seguridad para acceder a las Fístulas Arteriovenosas (FAVIs) de los pacientes, aunque compaginamos o agujas con sistemas de seguridad y 
agujas sin sistemas de seguridad en función de nuestro criterio profesional.

Son muchas las casas comerciales que tienen interés por crear una aguja que tenga un dispositivo de seguridad que sea del agrado de todos pero como no era ese nuestro propósito (buscar la "mejor" aguja con dispositivo de seguridad del mercado), hemos llevado a cabo nuestro estudio utilizando las agujas de la casa comercial que se había utilizado habitualmente.

\section{Objetivos}

- Evaluar las posibles diferencias entre las agujas con dispositivo de seguridad vs. las agujas sin dispositivo de seguridad para las FAVIs utilizadas en nuestros centros.

- Valorar y promover el uso de dispositivos de seguridad por los profesionales para evitar o reducir la siniestralidad laboral en el ámbito sanitario.

\section{Material y Método}

Se llevó a cabo un estudio prospectivo experimental con 42 pacientes portadores de una FAVI autóloga (descartamos prótesis). La muestra se redujo a 35 pacientes porque durante el estudio hubo 2 ingresos, 1 trasplante, 3 éxitus y 1 paciente decidió no participar en el estudio. El periodo de estudio estuvo comprendido entre los meses de enero a abril de 2007. Se realizaron 26 sesiones de diálisis, 13 con cada tipo de aguja.

El material utilizado fueron agujas con y sin sistema de seguridad del calibre 15 y $16 \mathrm{G}$ de la marca Nipro ${ }^{\circledR}$.

En el estudio se tuvieron en cuenta los siguientes ítems:

\section{Relacionados con el paciente}

- Tipo de acceso vascular

- Grado de dolor en las punciones (arterial y venosa)

- Complicaciones-Repunciones

- Sangrado intra/post HD

\section{Relacionados con la práctica profesional}

- Facilidad de uso

- Calibre de la aguja utilizado

- Flujo sanguíneo obtenido

- Presión venosa media obtenida

- Uso del sistema de seguridad

(en el caso de ser agujas con dicho sistema)

- Número de punciones

\section{Relacionados con el material}

- Registro de aquel material que fuera desechado o estuviese defectuoso

- Registro del funcionamiento o no del sistema de seguridad

La recogida de los datos se realizó en una plantilla elaborada específicamente para este trabajo (Anexo1). En la misma se especificaba el nombre del paciente y su acceso vascular. Para que el paciente pudiera valorar el dolor causado durante la punción, se utilizó una escala numérica del 0 al 5, mediante la cual el paciente designaba un número teniendo en cuenta la relación, $0=$ ausencia de dolor y $5=$ dolor insoportable. Hay que advertir muchos pacientes usan pomadas anestésicas con las que manifiestan no sentir ningún dolor durante la punción.

\section{Resultados}

Se analizaron un total de 35 pacientes ( 26 hombres) durante 13 sesiones con cada tipo de dispositivo, siendo un total de 455 sesiones las valoradas en cada caso.

La media de edad de los pacientes fue de 67,4 años. Los accesos vasculares valorados fueron 14 FAVIs radio - cefálicas, 14 húmero - cefálicas y 7 húmero - basílicas.

En cuanto al grado de dolor, tanto para la punción arterial como para la venosa, no hubo diferencias significativas en el uso o no del dispositivo de seguridad. Encontramos una media de 1,1 sin sistema de seguridad, frente a un 0,9 con sistema de seguridad para 
[ M. Brazalet Tejerina, et al ]

Estudio comparativo de agujas con sistema de seguridad vs agujas sin sistema de seguridad

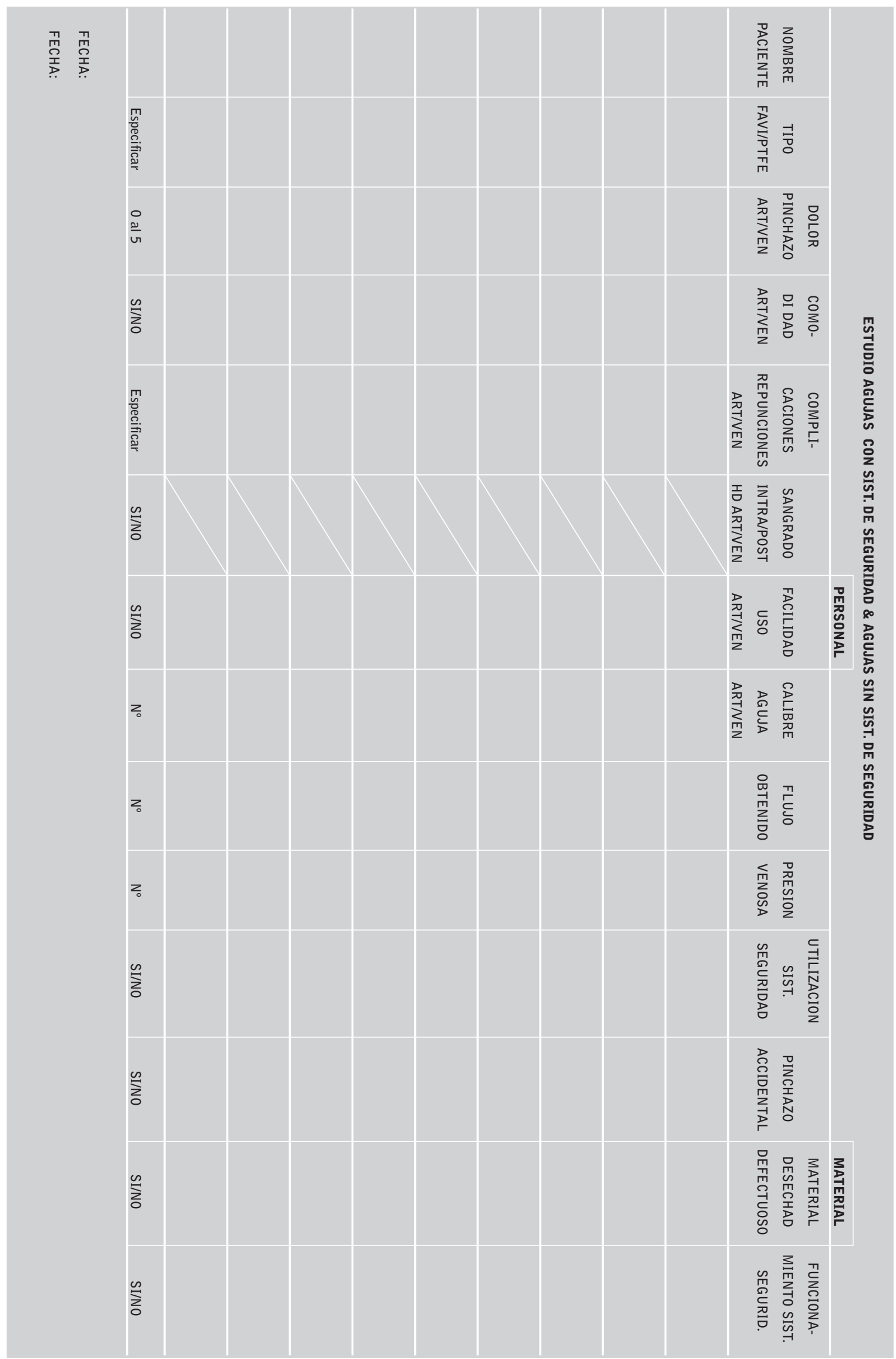


la arterial y una media de 1,3 vs 1,2 para la venosa (Fig.1).

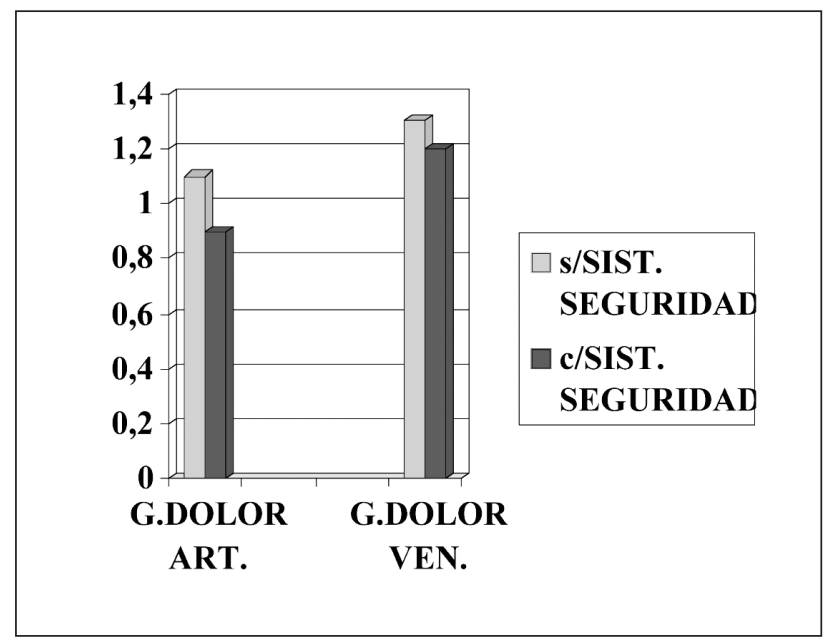

dispositivo de seguridad. Hemos registrado el número de veces que se producían estos eventos (Fig.3).

Durante el estudio no se produjo ningún pinchazo accidental y encontramos los siguientes problemas en la utilización de agujas con dispositivo de seguridad: de 910 punciones en 2 ocasiones se evidenció un uso incorrecto por haber olvidado la técnica, en 2 ocasiones no se pudieron utilizar debido a la proximidad de las dos agujas, que impedía maniobrar adecuadamente y en 24 ocasiones se atascó el dispositivo de seguridad. Cuando los dispositivos se atascaban, decidimos introducir la aguja en el contenedor de objetos punzantes y no comprobar si el material estaba defectuoso para evitar riesgos.

Figura 1. Valoración de la escala del dolor

Los flujos de sangre tampoco sufrieron variaciones significativas: $377 \mathrm{ml} / \mathrm{min}$ de media para agujas sin sistema de seguridad frente a $382 \mathrm{ml} / \mathrm{min}$ con sistema de seguridad. Lo mismo ocurre con las presiones venosas: una media de $170 \mathrm{mmHg}$ sin sistema de seguridad, frente a $172 \mathrm{mmHg}$ de media para las agujas con sistema de seguridad (Fig.2).

\begin{tabular}{|l|c|c|c|c|c|c|}
\hline & $\begin{array}{c}\text { repunción } \\
\text { arterial }\end{array}$ & $\begin{array}{c}\text { repunción } \\
\text { venosa }\end{array}$ & $\begin{array}{c}\text { sangrado } \\
\text { intraHD art }\end{array}$ & $\begin{array}{c}\text { sangrado } \\
\text { intraHD ven }\end{array}$ & $\begin{array}{c}\text { sangrado } \\
\text { postHD art. }\end{array}$ & $\begin{array}{c}\text { sangrado } \\
\text { postHD ven. }\end{array}$ \\
\hline $\begin{array}{l}\text { NO SIST. } \\
\text { SEGURIDAD }\end{array}$ & 2 & 6 & 10 & 10 & 5 & 16 \\
\hline SIST. & 1 & 4 & 12 & 7 & 10 & 4 \\
\hline SEGURIDAD & 1 & & & & & \\
\hline
\end{tabular}

Figura 3. Problemas detectados

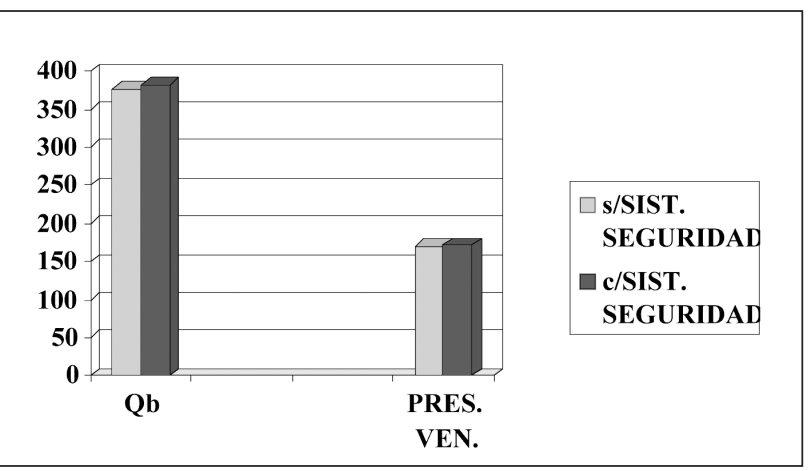

Figura 2. Flujos de sangre y presiones venosas

Tampoco se encontraron diferencias respecto a las repunciones, 0 sangrados intra 0 post HD. Apenas existieron dificultades a la hora de utilizar ambos tipos de agujas y se podría matizar que suelen ser los mismos pacientes los que presentan problemas de coagulación tanto con agujas con dispositivo como sin

\section{Discusión}

Aunque no hemos encontrado diferencias significativas entre el uso de agujas con o sin dispositivo de seguridad, con relación a los diferentes aspectos evaluados en el estudio es importante reseñar que:

No recomendaríamos este tipo de dispositivo para aquellas FAVIs recientes (con gran riesgo de extravasaciones y rasgados), porque aunque no ha sido demostrado en este trabajo, si hemos notado un mayor peso de la aguja, que podría rasgar este tipo de fístulas. También da problemas en aquellas que al ser tortuosas o con un cierto grado de inclinación, imposibilita su buen uso.

La zona rígida donde se alojará la aguja tras su uso es opaca y nos resultaría de mayor utilidad que fuera transparente en las ocasiones en las que hay que recolocar la misma, para ver si existe flujo de sangre. 
El uso del sistema de seguridad no se ha utilizado en varias ocasiones, ya sea por olvido, imposibilidad espacial o "atasco" del mismo. Creemos que a la hora intentar introducir la aguja en la zona rígida, podría haber sangre coagulada que impediría su desplazamiento. Este hecho no ha sido confirmado ya que, tras su uso infructuoso, se tiró al contenedor evitando una manipulación extra del mismo.

Para la activación del dispositivo de seguridad son necesarias ambas manos y creemos que sería deseable un dispositivo más sencillo en el que sólo fuera necesario utilizar una mano.

Es necesaria una mayor concienciación por parte del personal de enfermería para el uso de estos dispositivos de seguridad. Para ello creemos conveniente la creación y desarrollo de programas educativos, dirigidos al personal sanitario, con el que se promuevan las medidas adecuadas para su capacitación y formación en el uso de dispositivos y productos de seguridad, así como para la implantación de prácticas de trabajo más seguras.

Aunque nuestra muestra es limitada, la idea de tener que utilizar este tipo de agujas par FAVIs con dispositivos de seguridad de manera "obligada" ha hecho que nos mentalizásemos en su uso y nos hemos llevado una grata sorpresa porque no son tan "complicadas" como nos parecían en un principio.

\section{Conclusiones}

No hay diferencias remarcables en la utilización de agujas con dispositivos de seguridad respecto a las que no lo tienen y aunque su diseño puede mejorarse, es deseable su uso para evitar riesgos profesionales.

\section{Bibliografía}

1. Orden 82772005 , de 11 de mayo, de la Consejería de Sanidad y Consumo de la Comunidad de Madrid. BOMA. Pag 1582.

2. Ley $31 / 1995$, de 8 de noviembre, de Prevención de Riesgos Laborales.

3. Hernández MJ, Arribas JL, Misiego A, Gillamon I. "Estudio EPINETAC". Revista Rol de enfermería. 2006; 29 (2):94-98.

4. Montero R. Manzanares A. Escalas de valoración del dolor. Revista Jano. 2005; 68(1.553):41-44. 